\title{
Capillary supply in heavy-resistance trained non-postural human skeletal muscle
}

\author{
P. SCHANTZ
}

Department of Physiology III, Karolinska Institutet, S-11433 Stockholm, Sweden

In a previous study (Schantz 1982) it was shown that both the number of capillaries per muscle fibre and the fibre areas were to the same extent greater in heavy-resistance trained as compared to untrained postural muscle (vastus lateralis), thus resulting in the same number of capillaries per $\mathrm{mm}^{2}$. In order to investigate whether the increased number of capillaries per fibre might be due to the postural function and not the heavy-resistance training per se, the capillary supply in $\mathrm{m}$. triceps brachii of heavy-resistance and untrained subjects has been characterized and compared.

Subjects. 6 heavy-resistance trained male subjects (bodybuilders) volunteered to participate in this study. The means for their age, weight and height were 24 years, $96 \mathrm{~kg}$ and $1.84 \mathrm{~m}$, respectively. They had been training systematically for an average of 5 years. They trained $\mathrm{m}$. triceps brachii on the average 2.5 times per week, using 3 different exercises. The number of sets and repetitions per set were 3 and 7 , respectively. The weights were chosen so that the exertion should be maximal at the last repetition per set. All the subjects had been using anabolic steroids. For comparison, results from a study on endurance training of 6 subjects with untrained arms have been used (Schantz et al.). In the latter study, the arms were trained through skiing with backpack $30 \mathrm{~km}$ times 6 days/ week for 8 weeks.

Muscle biopsy sampling and analyses. Biopsies were obtained with the needle-biopsy technique (Bergström 1962) from the medial head of $\mathrm{m}$. triceps brachii at about $8 \mathrm{~cm}$ above the olecranon. Serial sections of the biopsies were stained for myofibrillar ATPase (Brooke \& Kaiser 1970) for identification of type I, IIA, IIB and intermediate (IB, IIC) fibres, as well as with the amylase-PAS method (Andersen 1975) for visualization of capillaries. Cross-sectional areas of the muscle fibres were measured from photos of the amylase-PAS sections (magnification $80 \times$ ) using an area measur- ing tablet (Apple Computer Inc., USA). The areas of $75 \pm 18$ type I and $76 \pm 27$ type IIA fibres were measured. (Type IIB and intermediate fibres were only seen to a very limited extent.) Capillary counts were performed as described by Andersen \& Henriksson (1977). Capillary density was calculated as the number of capillaries per $\mathrm{mm}^{2}$ of muscle crosssectional area $\left(\right.$ cap $\left.\times \mathrm{mm}^{-2}\right)$. The capillary per fibre ratio (cap $\times$ fibre $^{-1}$ ) was calculated as the total number of capillaries divided by the total number of fibres within a given area. The mean number of capillaries in contact with fibres of each type (CC) and CC per $1000 \mu \mathrm{m}^{2}$ of fibre type area were calculated by counting all the capillaries around each type I and type IIA fibre. Cap $\times \mathrm{mm}^{-2}$ was counted for an average cross-sectional area of $1.7 \pm 0.3 \mathrm{~mm}^{2}$.

Statistics. Results will be stated as means and standard error of the mean or range. The statistical significance of the differences between the heavyresistance trained and the untrained subjects was tested with Mann-Whitney's ranksum-test for unpaired data. Post-training values for the untrained subjects have been included in Fig. 1 for reason of comparison. However, no statistical significance has been tested using those values.

Results and discussion. The muscle fibre type distribution in $\mathrm{m}$. triceps brachii of the heavy-resistance trained subjects (HT) contained $49.2 \pm 9.7 \%$ type I, $49.8 \pm 9.7 \%$ type IIA, $0.5 \pm 0.3 \%$ type IIB and $0.5 \pm 0.2 \%$ intermediate fibres. The number of capillaries per fibre and capillaries around each fibre type did not differ between the heavy-resistance trained and the untrained subjects before training (UT) (Fig. 1, Table 1). Due to a rather great variation within both HT and UT, the only significant difference with respect to the remaining variables was in the number of capillaries around the type I fibres per $1000 \mu \mathrm{m}^{2}$ fibre area. However, a general tendency to a lower capillary supply per unit of muscle cross-sectional area in the HT sub- 
$\%$ of untrained subjects values

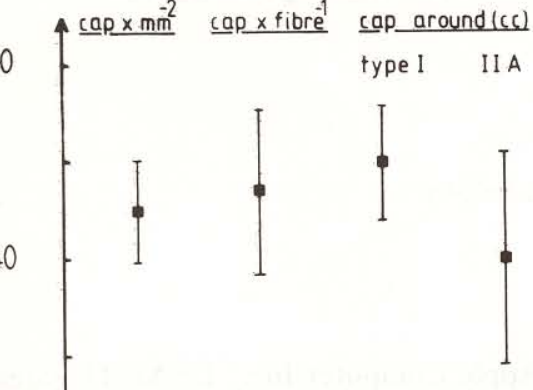

$\frac{c c \times \text { fibre area }}{\text { type I IIA fibre area }}$ type I IIA
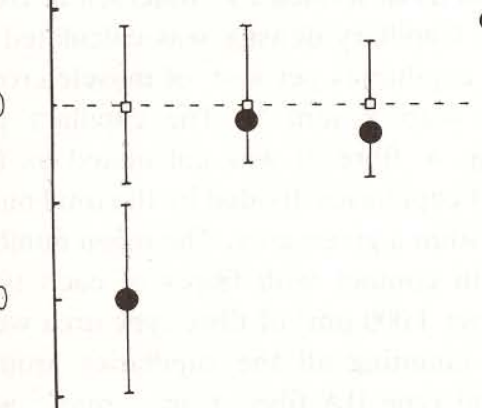

- heavy-resistance trained

- endurance trained 口 untrained (100\%)

Fig. 1. The capillary supply in the medial head of $\mathrm{m}$. triceps brachii, of heavy-resistance trained, endurance trained and untrained subjects. Significant differences between the untrained and heavy-resistance trained subjects are designated with an $a$ by the heavy-resistance trained subjects' symbols. No statistical evaluation has been performed using the values of the endurance trained. Values at the bottom of the figure are the absolute means for the untrained subjects, equivalent to the $100 \%$ level. The abbreviations for the different measures used to describe the capillary supply are explained in the text under "Muscle biopsy sampling and analyses". Values are given as means \pm SE. The SEs are expressed in per cent of the absolute mean.

jects seems clear. Furthermore, HT subjects with the greatest fibre areas also had the lowest capillary supply per unit area. However, it is possible that the capillary surface in contact with the fibres can increase through a more tortuous alignment of the capillaries and formation of capillary cross-bridges (Appell 1980). This possibility is unlikely since in the HT subjects no greater number of longitudinally cut capillaries was noted as compared to the untrained.

Table 1. Capillary supply in $m$. triceps brachii of heavy-resistance trained subjects (means and ranges)

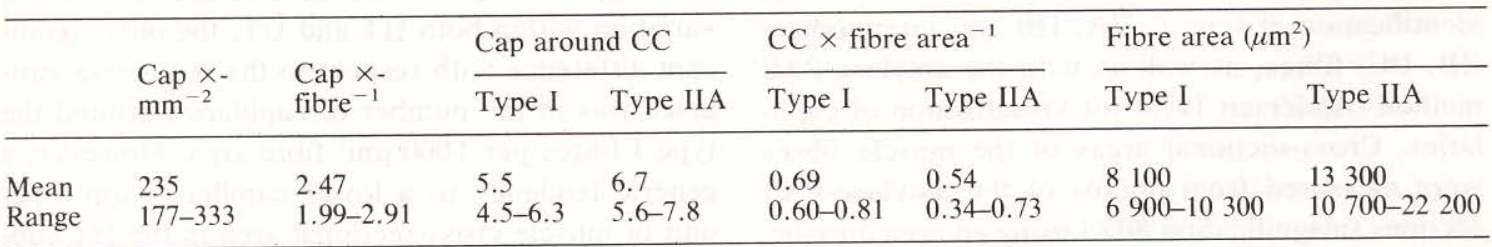


In conclusion, it seems as if heavy-resistance training per se does not stimulate to any capillary neoformation.

The author greatfully acknowledge A.-M. Gullström for secretarial assistance. The study was supported by grants from the Research Council of the Swedish Sports Federation.

\section{REFERENCES}

ANDERSEN, P. 1975. Capillary density in skeletal muscle of man. Acta Physiol Scand 95: 203-205.

ANDERSEN, P. \& HENRIKSSON, J. 1977. Capillary supply of the quadriceps femoris muscle of man: adaptive response to exercise. J Physiol 270: 677-690.
APPELL, H.-J. 1980. Morphological studies on skeletal muscle capillaries under conditions of high altitude training. Int J Sports Medicine 1: 103-109.

BERGSTRÖM, J. 1962. Muscle electrolytes in man. Scand J Clin Lab Invest Suppl. 68.

BROOKE, M. \& KAISER, K. 1970. Three "myosin ATPase" systems: The nature of their pH lability and sulfhydryl dependence. J Histochem Cytochem 18: 670-672.

SCHANTZ, P. 1982. Capillary supply in hypertrophied human skeletal muscle. Acta Physiol Scand 114: 635-637.

SCHANTZ, P., HENRIKSSON, J. \& JANSSON, E. Adaptation of human skeletal muscle to endurance training of long duration. Clin Physiol. Accepted for publication. 